\title{
Dentro de la fábrica de salchichas*1 2 : actores y proceso de toma de decisiones en el Parlamento Europeo
}

\author{
Inside the sausage factory: actors and decision-making \\ in the European Parliament
}

\author{
Nereo Peñalver García ${ }^{3}$ \\ Profesor Visitante en el Colegio de Europa (Brujas)
}

doi: $10.18543 /$ ced-55-2016pp145-163

Sumario: I. ¿Cómo co-legisla (de verdad) el Parlamento Europeo?

1. ¿Quiénes son los principales actores en una comisión parlamentaria?

2. ¿Cómo se prepara la legislación en el seno del PE? 3. Las negociaciones interinstitucionales PE-Consejo.-II. Nuevas dinámicas: ganadores y perdedores.-III. Nuevos retos, nuevas batallas.-IV. Referencias bibliográficas.

Resumen: Este artículo tiene por objeto ser un viaje a «la cocina» del Parlamento Europeo (PE). En él se explica como co-legisla el PE, desde que recibe una propuesta de la Comisión Europea, hasta su adopción. Pero más allá de presentar los pasos formales del «proceso legislativo ordinario», con el trabajo en comisión parlamentaria, las negociaciones con el Consejo y el voto en el pleno, el objetivo del presente estudio es dar una visión desde dentro de los actores, tanto oficiales como oficiosos, y las dinámicas que hacen posible la adopción de la legislación: el papel de los ponentes y los ponentes en la sombra, la mediación del Presidente para las propuestas legislativas sensibles, las coaliciones entre los grupos políticos,

* Recibido el 19 de mayo de 2016, aceptado el 21 de junio de 2016.

${ }^{1}$ Basada en la frase las leyes son como las salchichas: es mejor no ver como se hacen, atribuida al político alemán Otto von Bismarck, primer canciller de Alemania (1871-1890). Esta afirmación pone de manifiesto que legislar a veces implica «ingredientes desagradables» que es mejor no examinar.

2 Agradezco a Gabriel Álvarez Recarte y a David Chico, funcionarios del PE, sus comentarios a este artículo.

${ }^{3}$ Nereo Peñalver García es funcionario de la UE. Actualmente trabaja en la Comisión de Asuntos Exteriores del Parlamento Europeo y es Profesor visitante en el Máster sobre estudios políticos y administrativos europeos del Colegio de Europa (Brujas, Bélgica) donde da un curso sobre el Parlamento Europeo, nereo.penalvergarcia@ep.europa.eu

${ }^{4}$ Las opiniones expresadas en este articulo son exclusivamente las del autor y no representan necesariamente la posición oficial del Parlamento Europeo. 
o como intenta el Parlamento influir en la agenda legislativa pese a no tener el derecho de iniciativa legislativa, entre otros aspectos. En segundo lugar, el artículo presenta tres nuevas dinámicas dentro del PE y su efecto en términos de ganadores y perdedores en el seno de la Eurocámara. Finalmente, el artículo analiza los retos a los que se enfrenta el PE en esta nueva legislatura. Una legislatura presentada por el Presidente de la Comisión Europea, Jean Claude Juncker, como la de «la última oportunidad» y que va, sin duda, a determinar el futuro de la Unión.

Palabras clave: Parlamento Europeo, procedimiento de toma de decisiones, actores.

Abstract: This article is intended to be a trip to «the kitchen» of the European Parliament. It explains how the European Parliament co-legislates, since it receives a proposal from the European Commission to its adoption. But beyond presenting the formal steps of the "ordinary legislative procedure», with the work in committee, the negotiations with the Council and the vote in plenary, the objective of this study is to provide an insider's view of the actors, formal and informal, and the dynamics that make possible the adoption of legislation: the role of the rapporteurs and shadow rapporteurs, the mediation of President for sensitive legislative proposals, the coalitions between political groups, or how the Parliament attempts to influence the legislative agenda despite not having the right of legislative initiative ... among others. Secondly, the article presents three new dynamics within the EP (European Parliament) and its effect in terms of winners and losers within Parliament. Finally, the article analyzes the challenges facing the EP in this new term. A legislature qualified by the President of the European Commission, Jean Claude Juncker, as «the last chance» and that will, certainly, determine the future of the Union.

Keywords: European Parliament, decision-making process, actors.

\section{I. ¿Cómo co-legisla (de verdad) el Parlamento Europeo?}

Desde la entrada en vigor del Tratado de Lisboa, en diciembre 2009, la co-decisión se ha convertido en el procedimiento legislativo ordinario ${ }^{5}$ para adoptar las normas a nivel europeo. Prácticamente el $90 \%$ de las propuestas legislativas presentadas durante el mandato parlamentario precedente, 2009-14, siguieron el procedimiento de co-decision.

En el procedimiento legislativo ordinario, la Comisión Europea somete al Parlamento Europeo (PE) y al Consejo una iniciativa legislativa. Tras sus

${ }^{5}$ En este artículo utilizaremos co-decisión y procedimiento legislativo ordinario indistintamente aunque, según el Tratado (artículo 294 Tratado de Funcionamiento de la UE - TFUE - ), el término correcto es procedimiento legislativo ordinario. 
respectivos procedimientos internos, estas dos instituciones negocian, en pie de igualdad, para acordar un acto legislativo, es decir, una «ley europea» que puede ser un reglamento, una directiva o una decisión.

En su negociación bilateral, Parlamento y Consejo tienen tres rondas de negociación (cada una compuesta de múltiples reuniones) llamadas «lecturas» para llegar a un acuerdo. Si en estas tres lecturas las dos instituciones no llegan a un acuerdo, la propuesta de la Comisión se considerará no adoptada. En ese caso la Comisión puede someter una nueva propuesta legislativa.

Antes de negociar con el Consejo, el Parlamento debe adoptar su posición negociadora internamente. En este apartado explicaremos los procedimientos, actores y dinámicas que intervienen en la adopción, por el PE, de su posición negociadora.

El PE no dispone de iniciativa legislativa ${ }^{6}$, es decir, no puede proponer normas. La iniciativa legislativa es un cuasi-monopolio de la Comisión Europea, con algunas excepciones que permiten a un grupo de Estados Miembros, al Tribunal de Justicia o al Banco Central Europeo proponer legislación. Los Tratados dan también al Parlamento la potestad de invitar a la Comisión a presentar legislación en un ámbito en el que el PE considere que la UE debe legislar en vistas a aplicar los Tratados (artículo 225 TFEU). Para ello, la propuesta del PE debe recabar el apoyo de la mayoría de miembros que componen el PE, es decir, al menos 376 eurodiputados. Si la Comisión decide no presentar una propuesta, debe informar al Parlamento de sus razones.

En el caso general, como se ha indicado, la Comisión Europea presenta una iniciativa legislativa y la manda simultáneamente al Parlamento, al Consejo, a los parlamentos nacionales (que tienen ocho semanas para determinar si, en su opinión, la propuesta viola el principio de subsidiariedad), al Comité de las Regiones (CDR) y al Comité Económico y Social Europeo (CESE). La propuesta de la Comisión viene normalmente acompañada de un estudio del impacto económico, social y medioambiental de la propuesta en cuestión.

Cuando el PE recibe el proyecto de ley de la Comisión, el Presidente del Parlamento lo anuncia en el pleno y lo manda a la comisión parlamentaria competente en la materia para su estudio. Esta comisión parlamentaria pasa a ser la comisión competente para el fondo. Existen tres modalidades para asociar a otras comisiones parlamentarias al trabajo de la comisión competente para el fondo:

${ }^{6}$ Existen algunas excepciones a esta regla: El PE tiene iniciativa legislativa en algunos asuntos como la adopción del estatuto de los eurodiputados, la elección del defensor del pueblo europeo o el establecimiento de las disposiciones necesarias para hacer posible la elección de los eurodiputados por sufragio universal directo. 
1. Otras comisiones parlamentarias pueden emitir opiniones a la propuesta legislativa en sus ámbitos de competencia. Estas opiniones se traducen en propuestas de enmienda que son votadas por la comisión competente para el fondo. Si sus enmiendas son rechazas en el voto en la comisión competente para el fondo, la comisión que ha preparado la opinión no puede presentar enmiendas en el pleno.

2. Comisiones parlamentarias asociadas: cuando una parte de la propuesta legislativa de la Comisión forma parte del ámbito de competencias de otra comisión parlamentaria, la comisión competente para el fondo se ocupa de la mayor parte del informe y la comisión asociada prepara enmiendas a la parte de la propuesta legislativa bajo su ámbito de competencia. Estas enmiendas son, en principio, aceptadas por la comisión competente para el fondo sin votarlas. Las comisiones asociadas pueden presentar enmiendas en el pleno. Por ejemplo, la Comisión presentó, a inicios de la actual legislatura, una estrategia para el mercado interior digital. La comisión parlamentaria de mercado interior fue designada como competente para el fondo. Pero otras comisiones fueron asociadas para los ámbitos en los que eran competentes: la comisión de asuntos jurídicos se ocupó de la parte sobre copyright y propiedad intelectual, la comisión de libertades civiles trató en exclusividad los temas de protección de datos y lucha contra contenido ilegal en Internet, mientras que la comisión de educación y cultura fue responsable de la sección de la propuesta legislativa sobre los medios de comunicación.

3. Cuando el tema de la propuesta de la Comisión recae de modo indivisible en el ámbito de competencia de varias comisiones y el asunto reviste una importancia especial, se podrá decidir la aplicación del procedimiento de reuniones conjuntas de comisiones y votación conjunta. En este caso se prepara un único informe, con un ponente designado por cada una de las comisiones, el informe se debate en reuniones conjuntas de las dos comisiones y este es votado conjuntamente por las dos comisiones. Por ejemplo, la comisión de libertades civiles y asuntos de interior y la de control presupuestario adoptaron un informe con comisiones conjuntas sobre la protección de los intereses financieros de la Unión Europea (UE) en el derecho penal.

Este sistema responde al hecho de que, crecientemente, las propuestas de la Comisión cubren varios sectores, de manera que no se corresponden con la división de competencias entre las comisiones parlamentarias del PE. Las carteras de los Comisarios raramente se corresponden con las competencias de una sola comisión parlamentarias del PE. Por ejemplo tres co- 
misiones parlamentarias diferentes (asuntos jurídicos, medio ambiente y protección de los consumidores e igualdad de género) son competentes de la cartera de la Comisaria Jourová, que se ocupa de los asuntos de justicia, protección de los consumidores e igualdad de género.

El reparto de competencias de una propuesta legislativa entre varias comisiones se lleva a cabo en la Conferencia del Presidentes de Comisión (CPC). Este órgano político, integrado por todos los presidentes de comisiones parlamentarias, se ocupa de dirimir los conflictos de competencias entre comisiones. En caso de desacuerdo, el Presidente de la CPC somete una propuesta de reparto a la Conferencia de Presidentes (ver apartado 2 de este articulo).

\section{1. ¿Quiénes son los principales actores en una comisión parlamentaria?}

El/la presidente/a de comisión: preside las reuniones de comisión y representa a la comisión en el exterior (conferencias, visitas oficiales...) así como en las negociaciones con otras comisiones parlamentarias.

Cada comisión parlamentaria tiene cuatro vice-presidentes, cuya función es sustituir al presidente en sus tareas en caso de ausencia.

Cada grupo político (en el actual mandato del PE hay ocho grupos) tiene un portavoz, llamado coordinador, en cada comisión parlamentaria. Los eurodiputados no inscritos, esto es, que no están afiliados a ningún grupo político, no tienen coordinador que les represente. Los coordinadores deciden sobre los expertos que se van a invitar a las audiciones, designan a los ponentes de sus grupos, proponen informes de iniciativa en nombre de sus grupos, negocian la atribución de propuestas legislativas a su grupo e intentan mantener una posición coherente de su grupo en las negociaciones de los informes (proponen, por ejemplo, en que sentido debe votar el grupo una determinada propuesta legislativa).

Los ponentes. Una vez una propuesta legislativa de la CE es referida por el Presidente del Parlamento a una comisión parlamentaria, los coordinadores de los diversos grupos políticos interesados pujan, mediante un sistema de puntos, por que se les atribuya esta propuesta a su grupo. Una vez un grupo se hace con una propuesta legislativa, el coordinador escoge un ponente entre los miembros de su grupo en esa comisión parlamentaria. El/ la ponente será el responsable de negociar esa ley en nombre del PE. Los otros grupos políticos nombran los llamados ponentes en la sombra, que negociaran con el ponente la posición de la comisión parlamentaria en la materia.

El ponente guía la propuesta legislativa a través de la comisión parlamentaria y el pleno: presenta un proyecto de informe a la comisión parlamentaria (con las enmiendas que propone a la propuesta legislativa de la 
$\mathrm{CE}$; negocia las enmiendas a su proyecto de informe con los ponentes en la sombra de los otros grupos políticos; propone el sentido del voto a las enmiendas a su proyecto de informe durante el voto en comisión parlamentaria; presenta su informe en el pleno, antes de su voto. Asimismo, juega un papel fundamental en las negociaciones con el Consejo para la adopción de una propuesta legislativa.

Excepcionalmente, algunos proyectos legislativos sensibles tienen coponentes. Normalmente se da únicamente en el caso de propuestas de reformas institucionales, como la posición del PE ante la negociación de un nuevo Tratado o, más recientemente, la posición del PE en la reforma de la ley electoral de la UE. Los co-ponentes no pueden ser del mismo grupo político. Normalmente son de los dos principales grupos en la Eurocámara: Partido Popular Europeo (PPE) y Socialistas y Democrátas (S\&D).

Cada comisión parlamentaria debe ser representativa de las mayorías en el pleno. Es decir, si el grupo de los socialistas y demócratas (S\&D) tiene un 25\% de los eurodiputados en la Cámara, aproximadamente una cuarta parte de los eurodiputados de cada comisión deben ser del grupo S\&D.

Todos los eurodiputados pueden participar en las reuniones de todas las comisiones y presentar enmiendas a los textos que en ellas se debaten. Pero solo los miembros o los suplentes de una comisión parlamentaria pueden votar en esa comisión. En el caso de los suplentes solo pueden votar si se ausenta un miembro de pleno derecho de su mismo grupo político.

\section{2. ¿Cómo se prepara la legislación en el seno del PE?}

Una vez un ponente ha sido escogido como responsable de un proyecto legislativo se inicia la elaboración de su informe. El ponente puede usar varias fuentes para elaborar su informe: el secretariado de la comisión parlamentaria en cuestión le puede preparar un primer borrador de informe, que será modificado por el ponente con el asesoramiento de sus asistentes parlamentarios y del asesor en la materia de su grupo político. El ponente también puede pedir al servicio de estudios del PE un estudio en la materia. Por ejemplo, un estudio comparativo sobre la legislación actual en ese ámbito en los 28 Estados miembros de la UE. La comisión parlamentaria puede invitar a expertos en la materia a una audición parlamentaria para profundizar su conocimiento del asunto que se trate. La comisión también puede organizar intercambios de puntos de vista con otras instituciones de la UE como la CE, la presidencia del Consejo o el Tribunal de Cuentas.

Una vez finalizado, el ponente presenta su informe en comisión parlamentaria para una primera consideración por parte de los otros diputados. A partir de ahí se abre un plazo para enmiendas. Una vez recibidas todas las 
enmiendas, el ponente, con ayuda del secretariado de comisión, prepara enmiendas de compromiso entre todas las enmiendas recibidas y las negocia con los ponentes en la sombra. Una vez los compromisos adoptados, el ponente los presenta en una segunda consideración en comisión parlamentaria. Finalmente, se vota el informe en comisión y, en caso de ser aprobado (por mayoría simple de los votos emitidos), se manda al pleno para ser votado y adoptado como posición negociadora del PE.

¿Cómo se aseguran los líderes de los grupos políticos que sus diputados siguen sus consignas de voto? En el PE no existe, como en House of Cards, un Frank Underwood que tiene una lista con asuntos turbios de cada uno de sus diputados para chantajearles llegado el momento del voto. Los presidentes de los grupos utilizan tres métodos para garantizar la disciplina de voto: El primero son los debates en la reunión de grupo, donde se dan a conocer las diferentes sensibilidades y se adopta la posición del grupo para una determinada propuesta legislativa. En segundo lugar, cada grupo político recibe un tiempo, en función de su tamaño, para que sus diputados intervengan en el pleno. El Presidente del grupo tiene la prerrogativa de distribuir este tiempo de palabra en el pleno entre los diputados de su grupo, y puede utilizarlo como incentivo para que los eurodiputados díscolos voten con el grupo a riesgo de ser excluidos de trabajos parlamentarios futuros. Lo mismo ocurre con la distribución de informes por los coordinadores a nivel de comisión parlamentaria. Un diputado que no siga la línea del grupo tendrá más dificultades para ser ponente de un informe. Finalmente, el presidente del grupo político puede llamar al orden a un eurodiputado y reunirse con él para intentar convencerle de que vote con el grupo.

En el mandato parlamentario 2009-14, los liberales europeos (ALDE) tenían un gran poder. Al no tener ningún grupo mayoría parlamentaria, el voto de los liberales determinaba si ganaba el centro-derecha (PPE) o el centro-izquierda (S\&D). Las mayorías eran, pues, fluidas: en asuntos económicos los liberales de ALDE solían votar con el PPE, mientras que en temas relacionados con los derechos fundamentales y las libertades solían votar con la izquierda.

Esto ha cambiado al inicio del actual mandato parlamentario, en el que se observa un nuevo punto de ruptura: pro-integración europea vs anti-integración, a diferencia del anterior clivaje izquierda-derecha.

\section{Las negociaciones interinstitucionales PE-Consejo}

A la posición del PE votada en el pleno se le llama primera lectura. A partir de ahí empieza lo que llamamos el «ping pong» con el Consejo, con las dos instituciones negociando en hasta tres lecturas. 
En la práctica, las comisiones parlamentarias suelen decidir empezar negociaciones informales con el Consejo, en base al texto aprobado en comisión parlamentaria, antes de que el Parlamento adopte su posición en primera lectura en el pleno. Son los llamados «trilogos informales». La decisión para empezar las negociaciones informales con el Consejo debe ser respaldada por la mayoría los eurodiputados de la comisión parlamentaria.

El equipo negociador del PE está compuesto por el presidente de la comisión parlamentaria, el ponente y los ponentes en la sombra, estando así representados todos los grupos y sensibilidades políticas. El equipo negociador debe informar regularmente a todos los miembros de la comisión parlamentaria del progreso en las negociaciones.

A diferencia del equipo negociador del Parlamento, que está compuesto exclusivamente por políticos, el del Consejo puede estar presidido por el presidente del grupo de trabajo en cuestión (un alto funcionario). También puede presidirlo el presidente del Comité de Representantes Permanentes (COREPER), esto es el Embajador Representante Permanente ante la UE del país que ostenta la presidencia rotatoria del Consejo. Solo al final de las negociaciones suele el Consejo estar representado por un político, el Ministro del ramo del país que ostenta la presidencia rotatoria del Consejo.

El Consejo suele intentar sacar partido de su representación a nivel de funcionarios en las negociaciones. En primer lugar, usan su experiencia técnica como argumento en las negociaciones. En segundo lugar, al ser funcionarios, los negociadores del Consejo suelen decir que tienen un mandato del que no se pueden desviar, negando así el margen de maniobra necesario para acercarse a las posiciones del PE. Otro factor que desequilibra las negociaciones en favor del Consejo es que, dada la opacidad de sus negociaciones internas, el PE desconoce la posición individual de los diversos EEMM. Las reuniones de comisión del PE son públicas y se retransmiten en directo por Internet, de manera que el Consejo puede identificar aliados entre los eurodiputados. El PE, al contrario, desconoce que EEMM podrían apoyar sus posiciones. Se trata del eterno dilema entre la falta de transparencia (y el consiguiente margen de maniobra para ventaja del Consejo en la negociación), y el derecho de los ciudadanos a conocer como se elabora la legislación.

Cada presidencia del Consejo suele poner el énfasis en llegar a acuerdos en la legislación que es prioritaria para su país. Por lo tanto, en función de quien ostente la presidencia rotatoria, es más fácil o más complicado avanzar en ciertas negociaciones.

La Comisión Europea también está representada en las negociaciones, muy raramente a nivel político (Comisario). Normalmente suelen participar el jefe de la Unidad que ha elaborado la propuesta legislativa, su Director o el Director General. La Comisión tiene como papel dar un asesoramiento 
técnico e imparcial durante las negaciones y actuar como mediadora entre las partes.

En caso de llegar a tercera lectura (conciliación), el equipo negociador del PE pasa a estar presidido por un Vice-Presidente.

Una vez PE y Consejo llegan a un acuerdo, este es votado por el PE en comisión parlamentaria y en el pleno. En el caso del Consejo, el COREPER y el Consejo de Ministros deben aprobar el texto.

\section{Nuevas dinámicas: ganadores y perdedores}

En los últimos años se han instalado tres tendencias en el Parlamento Europeo. Cada una de ellas ha generado una nueva dinámica, con ganadores y perdedores.

La primera dinámica es la centralización del poder y la capacidad de influencia. En los últimos años se ha reforzado el papel y las competencias del Presidente, la Conferencia de Presidentes y la Mesa para garantizar la coordinación política interna ${ }^{7}$.

La Conferencia de Presidentes está compuesta por los líderes de los grupos políticos del PE y por un representante de los eurodiputados no inscritos (sin afiliación a un grupo político) seleccionado por el Presidente del PE. Entre sus competencias están la programación legislativa, establecer el proyecto de orden del día del pleno del Parlamento, las relaciones con las demás instituciones y órganos de la UE, con los parlamentos nacionales, y con terceros países e instituciones, determinar la composición y competencias de las comisiones, incluido el establecimiento de comisiones de investigación, y la autorización de los informes de propia iniciativa.

La Conferencia de Presidentes escoge las grandes líneas de trabajo institucionales y garantiza la defensa de los intereses del PE. Un ejemplo: Con la adopción del Tratado de Lisboa cambiaron los procedimientos mediante los cuales una ley europea es modificada o desarrollada, una vez se ha llegado a un acuerdo entre los co-legisladores. El Tratado de Lisboa distingue entre dos tipos de actos no-legislativos: actos delegados (artículo 290 TFUE) y actos de ejecución (artículo 291 TFUE).

Durante la legislatura pasada, 2009-2014, una de las grandes batallas entre el Consejo y el PE fue el determinar si un acto podía ser considerado

${ }^{7}$ Para un argumento más desarrollado sobre esta tendencia véase: Nathalie Brack, Olivier Costa y Clarissa Dri, «Le Parlement Europeen a la recherche de l'efficacite legislative: une analyse des evolutions de son organisation» Bruges Political Reserach Papers, n. ${ }^{\circ} 39$ (2015), p. 1-47. 
Delegado (con papel para el PE) o de Ejecución (con un papel muy limitado para el PE $)^{8}$.

Un ponente podría querer someter su informe a voto en el pleno, a pesar del debate entre PE y Consejo por determinar si un determinado acto de esa ley era un acto delegado o de ejecución. Sin embargo, la Conferencia de Presidentes prohibió la inscripción en el orden del día del pleno de toda legislación en disputa. Así, los líderes de los grupos políticos evitaron crear precedentes y minar la posición del Parlamento en las negociaciones con el Consejo en la materia.

La Mesa está compuesta por el Presidente, catorce Vice-Presidentes y cinco cuestores, que son miembros de la Mesa con voz pero sin voto. La Mesa es responsable de los asuntos económicos, de organización y administrativos que afecten a la organización interna del Parlamento, a los diputados, a la secretaría del PE y a sus órganos. En este sentido, las tareas más importantes de la Mesa son nombrar al Secretario General y a los altos cargos del PE (Directores y Directores Generales), establecer el anteproyecto de presupuesto del Parlamento, y adoptar la política inmobiliaria y de comunicación del Parlamento.

El papel del Presidente del Parlamento Europeo también ha cambiado desde la elección, a principios de 2012, del socialista alemán Martin Schulz que le ha dado un perfil más político y ha aumentado la visibilidad de la función. En algunas tradiciones parlamentarias, como la británica o canadiense, el Presidente del parlamento se limita a presidir el pleno y garantizar el respeto del reglamento interior de la Cámara. El papel del Presidente

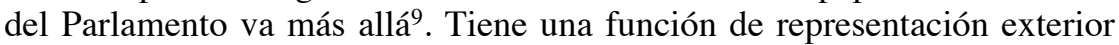
(con terceros países) y en las negociaciones con los Presidentes de las otras instituciones de la UE. También tiene prerrogativas en la implementación de los poderes legislativos y presupuestarios del Parlamento. Finalmente,

${ }^{8}$ En los actos delegados el PE puede controlar los poderes que los co-legisladores delegan a la CE para complementar o modificar ciertos elementos no esenciales de una ley europea. En este sentido, el PE y el Consejo, como co-legisladores, se pueden concentrar en determinar la orientación política y los objetivos de la ley, delegando en la CE la implementación detallada y a menudo técnica de la propuesta. Por ejemplo, actualizar una lista de productos en un anexo. El PE y el Consejo pueden revocar la delegación de poderes a la Comisión en caso de considerar que se ha excedido en sus funciones.

En el caso de los actos de ejecución, la Comisión se encarga de la aplicación de la legislación vigente, en concreto algunos actos jurídicamente vinculantes de la UE requieren condiciones uniformes de ejecución, en cuyo caso, se faculta a la Comisión para adoptar actos de ejecución. En estos casos el PE solo puede presentar sus objeciones al acto, pero la Comisión no tiene por qué aceptar la posición del parlamento.

9 Para un estudio en profundidad del papel del Presidente del Parlamento Europeo véase: Olivier Costa, «Le President du Parlement Europeen: un leader meconnu», Annuaire de droit de l'Union européenne 2013, (2015): 33-51. 
el Presidente del PE tiene un papel de dirección en la Asamblea, en su calidad de Presidente de las reuniones de la Conferencia de Presidentes y de la Mesa. Sin embargo, el Presidente continua teniendo un rol imparcial, centrado en la defensa del interés institucional, contrariamente al Presidente (speaker) de la Cámara de Representantes de Estados Unidos que es, a la vez, el líder de la mayoría parlamentaria en la Cámara, lo que le confiere un papel partidista.

Las sucesivas revisiones del reglamento interno del PE han aumentado los poderes del Presidente, sobre todo en lo que se refiere a sus capacidades durante los debates en el pleno. Según el reglamento, el Presidente «dispondrá de todos los poderes para presidir las deliberaciones del Parlamento y garantizar su desarrollo normal». Esto ha supuesto, entre otras medidas, el aumento de los poderes sancionadores del Presidente. El Presidente puede, por ejemplo, decidir sancionar a un eurodiputado sin someterlo al voto del pleno.

El PE ha de hacer frente a dosieres cada vez más técnicos, sobre todo en materia económica y de regulación financiera, mientras que los eurodiputados cuentan con menos tiempo para estudiarlos, dado el incremento de acuerdos en primera lectura (ver siguiente punto). En este contexto, el Presidente ha desarrollado un papel de mediación, sobre todo en temas legislativos sensibles, como las medidas de gobernanza económica, el Acuerdo Transatlántico de Comercio e Inversión Estados Unidos-UE (más conocido por sus siglas en inglés TTIP) o la protección de datos. El Presidente se reúne con el ponente, y los ponentes en la sombra, de los grandes informes legislativos para coordinar la posición institucional del PE.

En muchas comisiones los ponente y ponentes en la sombra desarrollan un esprit de corps y una visión que es representativa de la mayoría en esa comisión pero no de la mayoría parlamentaria. Imaginemos que el Parlamento está discutiendo una legislación sobre pesticidas. No es lo mismo si quien la debate es la comisión de agricultura, que será más favorable a su uso al pensar que ayudará a incrementar la producción agrícola, que si quien lo hace es la comisión de medioambiente, que adoptará una legislación más constringente. Normalmente este tipo de diferencias se resuelven en el pleno, donde todos los eurodiputados votan y, por consiguiente, todas las sensibilidades están representadas. Las enmiendas en el pleno, previas al voto pueden modificar la naturaleza de un informe (legislativo) haciéndolo más representativo de la mayoría parlamentaria. Sin embargo la implicación del Presidente durante las negociaciones, antes de llegar al voto en el pleno, garantiza que la posición adoptada respeta el interés de la institución y no solo la posición de una comisión parlamentaria en cuestión. 
Esta tendencia a la centralización del poder en el Presidente, la Mesa y la Conferencia de Presidentes, ha reforzado la influencia de los principales grupos políticos en detrimento del poder individual de los eurodiputados.

Los grupos de la mayoría pro-europea han incrementado legislatura tras legislatura el umbral para formar un grupo político y para poder influenciar en el pleno. En el caso de la formación de un grupo político, actualmente es necesario un mínimo de 25 eurodiputados provenientes de una cuarta parte de los Estados Miembros de la UE, es decir, siete países en la actualidad. Es sorprendente pensar que, hasta 1999, todos los eurodiputados de un grupo político podían provenir del mismo país. Respecto a las iniciativas en el pleno, para la mayoría de ellas, es necesario un grupo político o 40 eurodiputados. Por ejemplo, para presentar un candidato a Presidente del PE, para hacer enmiendas en el pleno, reenviar a la comisión parlamentaria correspondiente un texto que se va a votar en el pleno o solicitar una pregunta oral con debate en el pleno. Lo más probable es que esta tendencia no haga más que reforzarse. El aumento progresivo del número de diputados ha hecho necesarias nuevas reglas que no paralicen el Parlamento y lo actualicen. Además, el PE de 2019 será, probablemente, un Parlamento mucho más fragmentado políticamente y donde los grupos extremos del arco parlamentario tendrán una mayor representación. Eso llevara a los principales grupos pro-europeos a tomar decisiones en aras a incrementar los umbrales y dificultar que los eurodiputados euroescépticos puedan «sabotear» el trabajo de la mayoría parlamentaria.

Actualmente, además de su eventual función como ponente, la acción de los eurodiputados se limita, en gran mediad, al pleno. Un eurodiputado puede: 1) intervenir en el pleno durante un debate o durante la hora de preguntas a la Comisión, 2) intervenir en el pleno para rectificar/ aclarar una declaración previa, 3 ) intervenir en el pleno para realizar una explicación de voto (porque va a votar o ha votado en cierto sentido). Además de su actuación en el pleno, los eurodiputados pueden hacer preguntas escritas a la Comisión y el Consejo y presentar enmiendas.

En definitiva, los eurodiputados que no tienen un papel formal (Presidente, Vice-Presidente, líder de un grupo político, coordinador, ponente o ponente en la sombra) han visto su capacidad de influencia disminuida a lo largo del tiempo.

Es por eso que el papel de un ponente cuyo éxito le convertía en un miembro con una profunda influencia en el PE ya no se ha repetido desde que el éxito de Piet Dankert (socialista holandés), en la batalla con el Consejo por aumentar la influencia del PE, le llevó a dirigir el rechazo por el Parlamento del presupuesto de 1980 y le aupó, a hombros de los backbenchers, a la Presidencia del PE pese a existir un acuerdo entre los lí- 
deres de la Cámara para que el próximo presidente fuera el conservador alemán Egon A. Klepsch.

Esta racionalización en la organización y la toma de decisiones es fruto de la evolución lógica de un parlamento que empezó siendo una asamblea consultiva y hoy es ya un parlamento maduro, dotado de los principales atributos y poderes de un parlamento nacional ${ }^{10}$.

Sin embargo, pese a las crecientes limitaciones, cabe destacar que en la mayoría de los parlamentos nacionales los backbenchers tienen menos capacidad de influencia que en el PE. Además, como defiende la «Governing Theory» de Adler y Wilkerson ${ }^{11}$, en realidad, al reforzar el papel del PE los eurodiputados están reforzando, también, su imagen y su papel ante sus electores.

La segunda dinámica es la reducción en las propuestas legislativas (de la Comisión Europea) y la conclusión de mayores acuerdos en primera lectura. En los primeros dos años del actual mandato parlamentario, 20142016, las propuestas legislativas presentadas por la Comisión han disminuido en cerca de un 50\% comparado con el periodo 2009-2011: 200 en el mandato precedente para 112 en el actual. El inminente referéndum sobre el Brexit/ Bremain puede estar entre las causas de la disminución de la legislación propuesta por la Comisión, aunque no puede explicar por si sola este cambio de tendencia. De nuevo, los perdedores fruto de esta nueva realidad son los eurodiputados backbenchers puesto que al haber menos legislación tienen menos posibilidades de ser ponentes $\mathrm{y}$, por consiguiente, de influir en el PE.

En segundo lugar, el número de acuerdos en primera lectura ha aumentado progresivamente. Si bien en la legislatura 1999-2004 representaban el $28 \%$ de los acuerdos alcanzados, en el periodo 2004-2009 pasaron a representar el $72 \%$, posteriormente el $85 \%$ durante $2009-2014$ y en la legislatura actual representan ya un $80 \%$ de los acuerdos alcanzados.

Los datos nos indican que se negocia más tiempo informalmente pero, de ese modo, se llega a más acuerdos en primera lectura. La duración media para la adopción de una propuesta legislativa ha disminuido de 22 meses en la legislatura 1999-2004 a 18 meses en el periodo 2014-2016, mientras que el tiempo necesario para llegar a acuerdos en primea lectura ha aumentado, durante el mismo periodo, de 11 a 15 meses.

${ }^{10}$ Como dijo Josep Borrell, ex-Presidente del Parlamento Europeo (2004-2007) en el discurso de conclusión de su mandato, el Parlamento ya no es un tigre de papel. Disponible en: http://www.europarl.europa.eu/president/Presidents_old/president_borrell/speeches/es/files/ sp0137.htm Consultado el 30/04/2016.

11 E. Scott Adler y John D. Wilkerson, Congress and the politics of problem solving (Cambridge: Cambridge University press, 2013) 
Según Bressanelli, Koop y Reh, la adopción de acuerdos en primera lectura ha reforzado la cohesión del voto de PPE, S\&D y ALDE. Estos autores lo atribuyen a la pérdida de credibilidad que supondría para estos grupos el fracaso de adopción de una propuesta legislativa en primera lectura después de haber llegado a un compromiso informal con el Consejo, poniendo en cuestión llegar a acuerdos en futuras reuniones. Esta pérdida de credibilidad de los eurodiputados de estos grupos seria más preocupante si cabe dado que estos grupos políticos están también representados al otro lado de la mesa, en muchos de los gobiernos negociando en nombre del Consejo $^{12}$.

Muchos eurodiputados están descontentos con la creciente conclusión de acuerdos en primera lectura. Esta situación no les da suficiente tiempo para informarse sobre acuerdos importantes negociados en otras comisiones parlamentarias de las que no son miembros. Una de las posibles soluciones a esta situación ha sido establecer la posibilidad en el reglamento interno del PE de que el equipo negociador del PE tenga que recibir su mandato negociador del pleno, en lugar de que este se base en el texto adoptado en comisión parlamentaria.

La tercera dinámica es la creciente influencia del Consejo Europeo en la legislación adoptada. Según los Tratados ${ }^{13}$, el Consejo Europeo es la institución compuesta por los jefes de Estado y de gobierno de los EEMM de la UE, cuya función es dar el impulso necesario para el desarrollo de la UE y definir sus orientaciones y prioridades políticas generales. Pero desde el inicio de la crisis económica y financiera, el Consejo Europeo ha influido crecientemente la legislación. Lo ha hecho mediante la publicación detallada, en las conclusiones de sus reuniones, de la posición del Consejo, algo que compete al Consejo de Ministros, no a los jefes de Estado y de gobierno, restando por consiguiente la capacidad de negociación de aquellos. Algunos ejemplos claros fueron las negociaciones para la adopción de la patente europea o las negociaciones sobre la Política Agrícola Común (PAC) en el marco de las discusiones sobre el Marco Financiero Plurianual (MFP) para 2014-20. El perdedor de esta nueva dinámica es el PE puesto que, para la mayoría de eurodiputados, es muy difícil oponerse a lo que deciden los máximos líderes de sus países, sobre todo si el partido del eurodiputado en cuestión está en el gobierno.

12 Edoardo Bressanelli, Christel Koop y Christine Reh, «The impact of informalisation: early agreements and voting cohesión in the European Parliament», European Union Politics, n. ${ }^{\circ} 17: 1$ (2016), 91-113.

13 Artículos 13 y 15 del Tratado de la Unión Europea (TUE). 


\section{Nuevos retos, nuevas batallas}

Hasta ahora las batallas que ha presentado el Parlamento han tenido como objeto, fundamentalmente, el acrecentar sus poderes ${ }^{14}$. El PE ha reforzado sus poderes por dos vías: formales e informales. Desde el punto de vista formal cabe señalar las siguientes líneas:

En sus inicios el PE tenía tres prerrogativas principales: La primera era la posibilidad de recibir y debatir un informe anual de actividades de la Comisión. La segunda, el derecho a recibir respuestas de la Comisión a las preguntas parlamentarias (orales y escritas). Finalmente, el Parlamento podía presentar una moción de censura a la Comisión, una opción que requería $2 / 3$ de los votos, y que votaran al menos la mitad de miembros que componen el Parlamento.

Desde las negociaciones del Tratado de Ámsterdam el Parlamento ha estado presente en la Conferencias intergubernamental como observador. En paralelo, el PE fue progresivamente aumentando sus poderes presupuestarios y legislativos mediante los sucesivos Tratados: procedimiento de consulta (Tratado de Roma, 1957), procedimientos de cooperación y de dictamen conforme (Acta Única Europea, 1986), co-decisión (Maastricht, 1992), extensión de los ámbitos a los que se aplica la co-decisión en Ámsterdam y Lisboa, entre otros.

Por su parte, el refuerzo de los poderes informales se ha llevado a cabo por tres vías:

En primer lugar la estrategia llamada de foot in the door o pie en la puerta. Es decir, el uso de competencias en un ámbito para presionar y ganar influencia en otro. Todo empezó con los poderes presupuestarios. En 1975 los EEMM hicieron del PE la co-autoridad presupuestaria, junto con el Consejo. El Parlamento utilizó estos poderes para presionar al Consejo y ganar competencias legislativas y, posteriormente, de control del ejecutivo.

La segunda estrategia fue la inclusión en el reglamento del PE de prácticas, como resultado de una interpretación muy flexible de los Tratados, que posteriormente se daban como fait accompli y, a menudo, se codificaban en el siguiente Tratado o acuerdo interinstitucional. Por ejemplo, la inscripción de las audiciones de los Comisarios.

La tercera estrategia ha consistido en la conclusión de acuerdos interinstitucionales entre el Parlamento y la Comisión. Todo empezó en 1990, con la redacción de un código de conducta para las relaciones entre estas instituciones. El acuerdo interinstitucional actual, llamado Acuerdo Marco,

14 Para más información sobre las principales batallas del Parlamento para acrecentar sus poderes ver: Julian Priestley, Six battles that shaped Europe's parliament, (London: John Harper, 2008) 
es de 2010, y cubre los ámbitos siguientes: 1) Rendición de cuentas de la Comisión ante el Parlamento: el Presidente de la Comisión es elegido por el $\mathrm{PE}$, y el Colegio de Comisarios es también votado por el PE después de someterse los Comisarios a audiciones en las diversas comisiones parlamentarias. Además, los Comisarios rinden cuentas periódicamente ante el PE, ya sea en comisión parlamentaria o en el pleno; 2) acceso de los eurodiputados a la información, sobre todo a mandatos de negociación y al contenido de las negociaciones previamente a la conclusión de acuerdos internacionales; 3) cooperación legislativa y participación del PE en la elaboración del programa anual de la Comisión; 4) participación del PE en las reuniones con expertos nacionales (antigua comitología).

Así, el Parlamento actual ya dispone prácticamente de las competencias propias de los parlamentos nacionales. Es co-legislador y adopta el presupuesto junto con el Consejo, aprueba a la Comisión y la somete a control parlamentario durante su mandato.

A continuación, exponemos algunos de los retos a los que tendrá que hacer frente el PE durante la presente legislatura:

1. ¿Cómo puede la mayoría parlamentaria que apoya al primer Presidente de la Comisión surgido del proceso de Spizenkandidaten seguir manteniendo el control parlamentario del ejecutivo? Por primera vez, en las elecciones europeas de mayo 2014, los principales partidos políticos europeos presentaron candidatos a Presidente de la Comisión Europea. Este proceso se llamó Spizenkandidaten, de la palabra alemana para designar al cabeza de lista en unas elecciones ${ }^{15}$. La mayoría de los líderes de los grupos políticos en la Eurocámara (excepto los conservadores británicos del ECR y los euroescépticos del EFD) apoyaron que el próximo Presidente de la Comisión fuera el candidato del partido capaz de conseguir el apoyo de una mayoría parlamentaria. Juncker fue el elegido como candidato del partido que ganó las elecciones al PE, para intentar reunir una mayoría parlamentaria en torno a su programa. Lo consiguió y fue el primer Presidente de la Comisión elegido por el PE después de la entrada en vigor del Tratado de Lisboa ${ }^{16}$. El PE ve al

15 Para un análisis detallado sobre la primera campaña presidencial pan-europea véase: Nereo Peñalver y Julian Priestley, The making of a European President (Hampshire: Pallgrave Macmillan, 2015)

${ }^{16}$ El articulo 17 (7) del Tratado de la Unión Europea reza: «Teniendo en cuenta las elecciones al Parlamento Europeo y tras mantener las consultas apropiadas, el Consejo Europeo propondrá al Parlamento Europeo, por mayoría cualificada, un candidato al cargo de Presidente de la Comisión. El Parlamento Europeo elegirá al candidato por mayoría de los miembros que lo componen» (énfasis añadido). 
Presidente surgido del proceso de Spizenkandidaten como su Presidente. La manera en que se interpretó el Tratado dio nuevos poderes al PE a los que no quiere renunciar. El fracaso de la Comisión Juncker podría resultar en un replanteamiento del método por el cual se le eligió. Por eso, por primera vez, hay una mayoría parlamentaria que apoya las iniciativas de la Comisión Juncker. Esta mayoría está compuesta por PPE, S\&D y ALDE. Esto explica, por ejemplo, porque después del escándalo de Luxleaks no hubo una moción de censura contra la Comisión Juncker. Esta situación podría dificultar en cierta medida la tarea del PE. En una legislatura en la que hay muchas menos propuestas legislativas, lo normal sería que los eurodiputados se centraran en las tareas de control del ejecutivo. Sin embargo, la existencia de una mayoría parlamentaria que apoya a la Comisión dificulta esa tarea.

2. La batalla por influir durante todo el ciclo del proceso legislativo. Mediante el incremento de sus poderes presupuestarios y la extensión de las áreas (bases jurídicas del Tratado) en las que el PE es co-legislador, el Parlamento ha consolidado su papel en este sentido. Actualmente el foco de atención ha evolucionado. En primer lugar, a ¿cómo garantizar la calidad de la legislación adoptada por el PE? En segundo lugar, el Parlamento quiere extender su influencia a todas las fases del ciclo legislativo, es decir, a la adopción de las prioridades legislativas de la Comisión, a las consultas pre-legislativas y al control parlamentario de la aplicación por los EEMM de la legislación adoptada.

Tradicionalmente el PE ha intentado influenciar la agenda legislativa de la Comisión Europea. Lo ha hecho de dos maneras: mediante informes de iniciativa, en los que el PE pide a la Comisión Europea legislar en un cierto ámbito y expone su posición en la materia, indicando también el sentido de la regulación. Por otro lado, también lo ha hecho utilizando las audiciones a los Comisarios antes de la investidura de la Comisión Europea, y las negociaciones con el Presidente de la Comisión antes de que este tome posesión del cargo, para así influir en las prioridades de la Comisión Europea para su mandato de cinco años. Como consecuencia del acuerdo interinstitucional «Legislar mejor», aprobado recientemente, el PE y el Consejo determinan ahora, conjuntamente con la Comisión las prioridades legislativas de la UE.

El Tribunal de Cuentas elabora informes periódicos sobre el impacto de una determinada ley europea en los EEMM, así como el impacto de los programas de apoyo financiero de la UE. El PE quiere utilizar esta información en la fase de consultas pre-legisla- 
tivas cuando esté enmendando legislación para que los cambios tomen en consideración los problemas surgidos durante la aplicación de la legislación.

Finalmente, el PE quiere hacer un seguimiento de la implementación por los EEMM, de la legislación adoptada a nivel europeo. ¿Cómo asegurarse que los EEMM no dificultan la transposición de la legislación europea, haciéndola más compleja, mediante el denominado gold-platting? Este factor perjudica a menudo la imagen de la UE, vista como un ente burocrático, cuando en realidad son los EEMM los que añaden, en gran medida, burocracia al transponer la legislación europea al ordenamiento jurídico nacional.

3. El reto de influir una legislación cada vez más técnica. Los EEMM representados en el Consejo tienen ejércitos de funcionarios detrás de sí, trabajando en los Ministerios. ¿Cómo puede el Parlamento post-Lisboa, que es prácticamente total co-legislador con el Consejo, continuar teniendo la expertise necesaria para ejercer sus derechos en dosieres cada vez más técnicos?

4. La batalla por reanudar el vínculo con los electores. Una de las prioridades de los actuales líderes de la UE, tanto en la Comisión como en el PE, es recobrar la confianza de los ciudadanos en el proyecto europeo. Para ello, es necesario mejorar la imagen de la institución y de su utilidad en beneficio de los votantes. ¿Cómo acercar más a los ciudadanos el trabajo parlamentario?

5. La disyuntiva entre eficacia vs. transparencia y rendición de cuentas. Legislar a 28 es complicado. Los negociadores necesitan espacio para negociar y llegar a acuerdos teniendo en cuenta las diversas sensibilidades políticas y geográficas. Por eso, antes de los trílogos, actualmente ya se llevan a cabo «reuniones preparatorias técnicas», en las que el secretariado del PE, los asesores de los grupos políticos, el responsable del dossier en la Comisión Europea y un miembro del grupo de trabajo en la materia en el Consejo, se reúnen para acercar posiciones.

La actual Defensora del Pueblo europea, la irlandesa Emily O'Reilly, ha abierto una investigación sobre la transparencia de los trílogos en aras de una adopción de legislación más transparente en el seno de la UE. ¿Qué es más importante, la celeridad con la que se adopte la legislación o su transparencia? En caso de mayor transparencia de los trílogos, probablemente aumentaría el papel, el nivel y el contenido de las reuniones preparatorias técnicas.

En definitiva, ¿debe el PE ser una fábrica de salchichas cuyos ingredientes no queremos ver? o ¿debe ser una institución transparente? 
La capacidad del PE de responder a estos desafíos durante la presente legislatura determinará el que, como dijo el Presidente Juncker, los ciudadanos le den otra oportunidad a la UE.

\section{Referencias bibliográficas}

Adler, E. Scott y John D. Wilkerson. Congress and the politics of problem solving. Cambridge: Cambridge University press, 2013.

Brack, Nathalie, Olivier Costa y Clarissa Dri, «Le Parlement Europeen a la recherche de l'efficacite legislative: une analyse des evolutions de son organisation». Bruges Political Reserach Papers, n. ${ }^{\circ} .39$ (2015): 1-47.

Bressanelli, Edoardo, Christel Koop y Christine Reh. «The impact of informalisation: early agreements and voting cohesión in the European Parliament», European Union Politics, n. ${ }^{\circ}$ 17:1 (2016), 91-113.

Costa, Oliver, «Le President du Parlement Europeen: un leader meconnu», Annuaire de droit de l'Union européenne 2013, (2015): 33-51.

Peñalver, Nereo y Julian Priestley. The making of a European President. Hampshire: Pallgrave Macmillan, 2015.

Priestley, Julian. Six battles that shaped Europe's parliament. London: John Harper, 2008. 


\section{Derechos de autor (Copyright)}

Los derechos de autor (distribución, comunicación pública, reproducción e inclusión en bases de datos de indexación y repositorios institucionales) de esta publicación pertenecen a la editorial Universidad de Deusto. El acceso al contenido digital de cualquier número de Cuadernos Europeos de Deusto (CED) es gratuito, transcurridos 6 meses desde su publicación. Los trabajos podrán descargarse, copiar y difundir, sin fines comerciales y según lo previsto por la ley. Así mismo, los trabajos editados en CED pueden ser publicados con posterioridad en otros medios o revistas, siempre que el autor indique con claridad y en la primera nota a pie de página que el trabajo se publicó por primera vez en CED, con indicación del número, año, páginas y DOI (si procede). 